\title{
A Fault-tolerant Mutual Exclusion Algorithm in Asynchronous Distributed Systems
}

\author{
Yoon Kim \\ Dept. of Computer Information and Security \\ Korea National College of Welfare, Pyeongtaek, Korea
}

\begin{abstract}
Mutual Exclusion is one of the most studied topics in distributed systems where processes communicate by asynchronous message passing. It is often necessary for multiple processes at different sites to access a shared resource or data called a critical section (CS) in distributed systems. A number of algorithms have been proposed to solve the mutual exclusion problem in distributed systems. In this paper, we propose the new algorithm which is modified from Garg's algorithm[1] thus works properly in a fault-tolerant system. In our algorithm, after electing the token generator, the elected process generates a new token based on the information of the myreqlist which is kept by every process and the reqdone which is received during election. Consequently, proposed algorithm tolerates any number of process failures and also does even when only one process is alive.
\end{abstract}

Keyword: Mutual Exclusion, Critical Section, Fault-Tolerant, Fault Detection, timeout, safety, liveness, fairness

\section{INTRODUCTION}

Mutual Exclusion has been one of the fundamental issues in distributed systems. It is often necessary for multiple processes at different sites to access a shared resource or data called a critical section (CS) in distributed systems. The problem is to ensure that only one process is allowed to enter the critical section at a time. A number of algorithms have been proposed to solve the mutual exclusion problem in distributed systems. These algorithms are mainly divided into two groups: permission-based and token-based.

In permission-based algorithms, a process may enter the critical section after receiving permissions from all other processes. In token-based algorithms, a unique token (also known as a PRIVILEGE message) is shared among all processes and only one process can enter its critical section if it possesses the token. Examples of permission-based algorithms are Agrawal-Abbadi's algorithm[2], Ricart- Agrawala's algorithm[3], Singhal's algorithm[4] and Maekawa's algorithm[5]. Examples of tokenbased algorithms are Susuki-Kasami's broadcast algorithm[6], Singhal's heuristic algorithm[7], Mamum's Group algorithm[8] and Raymond's tree based algorithm[9] .

Few fault-tolerant token-based algorithms $[10,11,12,13]$ have been proposed to handle node failures and message loss. Mishra and Srimani algorithm[11] uses the concept of circulating a privilege message (token) among the sites. Nodes

\footnotetext{
* Corresponding author,Email : ykim@hanrw.ac.kr Manuscript received Aug. 10, 2012; revised Sep 17, 2012; accepted Sep 27, 2012
}

use timeout and probe messages to detect failure of the current token holder.

In this paper, we present a new fault-tolerant distributed mutual exclusion extension for Garg's decentralized tokenbased mutual exclusion algorithm in distributed system which assumes a fault-free system. Garg's Algorithm works properly in fault-free systems. However, it does not tolerate failure of a process, especially a token holding process or a process that has requested the token, which results in the token loss in the entire system.

In this case, a system is in deadlock because any request does not arrive from a process holding the token and thus any process can not enter the critical section. This does not satisfy liveness property of mutual exclusion, that every request for the critical section is eventually granted. We also have implemented the solution of this problem in this paper. Consequently, it is designed to perform properly even when any of the cooperating processes in the system are malfunctioned. That is, the algorithm designed in this paper tolerates any number of process failures even in case that only one process is alive in the system.

\section{GARG'S ALGORITHM}

To request the critical section, a process increases its component in $v$ vector and sends the request with its $v$ vector to all the processes. If a process has the token, then it checks for eligible requests and sends the token to one of the eligible processes. On receiving the token, a process is eligible to enter the critical section. To release the critical section, it sends the 
token to the next eligible process or if there's no eligible process, it holds the token until it receives a request. To ensure fairness, all the processes piggyback $v$ vector on all outgoing messages.

When a process receives a program message, it updates its old value in $v$ vector. To solve the problem that a request broadcast may never reach the token if it was made when the token was in transit between two processes, each process maintains myreqlist. Whenever the token is received, it is updated with all the entries in the myreqlist. This algorithm requires, at most, $\mathrm{N}$ messages per critical section invocation: $(\mathrm{N}-1)$ request messages and one token message.

\section{PROPOSED ISSUES}

We have modified the Garg's algorithm in order to make it also work properly in a fault-tolerant system. In this section, we discuss the problems of Garg's algorithm in terms of process failures and present the changes needed to make the Garg's algorithm fault tolerant to these failures.

\subsection{Process Failures}

Processes may fail by crashing. Crashed processes nay stop their execution permanently or recover from failures restarting execution. In this paper, we focus on only the former case called fail-stop failures. Since we assume that the communication link is reliable, we do not consider the link failures at this time. In addition, we distinguish crashed processes into the following two cases.

- A process with the token (token holder) fails.

- A process that has requested the token fails.

\subsection{Fault Detection}

To detect process failures, we use Acknowledgements and timeout-based re- transmission of messages which are commonly used techniques for fault detection in a distributed system. In our algorithm, a process detects process failures in the following cases.

First case is that process does not receive message that it is using the token now from the token holder while waiting for the token periodically. Second case is that process does not receive an acknowledgement from the process that will receive the token after it asks aliveness before sending the token.

\subsection{Fault-Tolerance}

Our algorithm is fault-tolerant because a process detects above two cases of process failures and takes an action as follows:

1) Process Failure Case 1: When a process detects the failure of the token holder, it starts an election algorithm to elect a token generator. The token generator sends the token after checking the next eligible process.

2) Process Failure Case 2: Before a process sends the token, it asks the receiving process' aliveness. If it does not receive an acknowledgment within a timeout period, it detects the process failure and checks again for the next eligible process to send the token.

\section{SPECIFICATION OF FAULT-TOLERANT ALGORITHM}

In this section, we propose our own fault tolerant extension to the original Garg's algorithm[1]. We consider a distributed system consisting of $\mathrm{N}$ processes denoted by $\left\{\mathrm{P}_{1}, \mathrm{P}_{2}, \ldots, \mathrm{P}_{\mathrm{n}}\right\}$, which are fully connected, communicating only by message passing. The system is synchronous i.e. both process speed and message transmission times are bounded. Processes can fail only by crashing, and crashes are permanent.

\subsection{Basic Idea}

The rules of our algorithm are as follows:

To request the critical section, a process increments its component in $v$ vector and sends the request with its $v$ vector to all the processes.

On receiving a request message, a process updates its old value in $v$ vector and appends the request in the list (myreqlist). If it has the token and does not use the critical section, then it checks for eligible requests and sends the token to one of the eligible processes. But if it is in the critical section, it sends "token_using" message to the requesting process.

On receiving the token, a process is eligible to enter the critical section and sends back an acknowledgment. To release the critical section, a process checks for eligible requests in the list (myreqlist) and sends the token to one of the eligible processes. If there is no eligible process to send the token, the process holds the token.

When a process receives a request, it sends a "token_using" message to the requesting process if it has the token and is in the critical section. The token holding process periodically sends the "token_using" message to the processes in the myreqlest.

After a process sends a request, if it does not receive the token and the "token_using" message within a timeout, it suspects token loss due to the token holding process failure. Then, it sends a "token_loss_detect' message to all other processes to initiate an election process. After a process sends the token to the next eligible process, if it does not receive an acknowledgement within a timeout period, it suspects the process failure. Then, it checks the eligible process and sends a newly generated token.

In our algorithm, after electing the token generator, the elected process generates a new token based on the information of the myreqlist which is kept by every process and the reqdone which is received during election. To ensure fairness, all the processes piggyback $v$ vector on all outgoing messages. When a process receives a program message, it updates its old value in $v$ vector.

\subsection{System Model}

The model of our algorithm is an asynchronous system composed of a set of $\mathrm{N}$ processes denoted by $\mathrm{P}_{1}, \mathrm{P}_{2}, \ldots, \mathrm{P}_{\mathrm{n}}$. The processes are fully connected without any shared memory or a global clock and communicate only through message passing. Further assumptions on the message delay and system failures are as follows: 
(1) Message Delay : Message delay is unbounded since the algorithm is based on an asynchronous model.

(2) Communication Links : Communication links are bidirectional and reliable, that is, no message is lost and changed.

(3) System Failures : There are no faults in the system, that is, processes are reliable.

(4)Token Loss : Since the processes and communication links are reliable, the token loss does not happen.

(5) Timeout : We use timeout as a timer to detect the token loss.

\subsection{Data Structures}

Each process Pi has variables described in Fig. 1.

\begin{tabular}{|c|c|}
\hline$v$ & $\begin{array}{l}\text { An array of integers. } v[\mathrm{i}] \text { represents the } \\
\text { number of request of } \mathrm{Pi} \text {. } \\
\text { Initially, } v[\mathrm{i}]=0 \text { for } \mathrm{Pi}(1=\mathrm{i}=\mathrm{N}) \text {. }\end{array}$ \\
\hline myreqlist & $\begin{array}{l}\text { A list of (pid, reqvector). It denotes some } \\
\text { request that has not been fulfilled yet. When } \\
\mathrm{Pi} \text { receives a request, it appends the request } \\
\text { to the list. The list is used to update the } \\
\text { token. }\end{array}$ \\
\hline inCS & $\begin{array}{l}\text { A boolean variable which is set to true when } \\
\mathrm{Pi} \text { is in the critical section. }\end{array}$ \\
\hline have & $\begin{array}{l}\text { A boolean variable which is set to true when } \\
\mathrm{Pi} \text { receives the token and is set to false when } \\
\mathrm{Pi} \text { releases the token. The token has the } \\
\text { following variables. Any process holding the } \\
\text { token does the computation using these two } \\
\text { variables }\end{array}$ \\
\hline reqdone & $\begin{array}{l}\text { An array of integers. reqdone }[\mathrm{i}] \text { equals the } \\
\text { number of requests made by } \mathrm{Pi} \text { that has been } \\
\text { fulfilled. Initially, reqdone }[\mathrm{i}]=0 \text { for } \mathrm{Pi}(1=\mathrm{i} \\
=\mathrm{N}) \text {. }\end{array}$ \\
\hline reqlist & $\begin{array}{l}\text { A list of (pid, reqvector). It denotes some } \\
\text { request that has not been fulfilled yet. For the } \\
\text { fault-tolerance, two more variables are used. }\end{array}$ \\
\hline timeout & A timer for the detection of token loss. \\
\hline flag & $\begin{array}{l}\text { A string variable which is set to } \\
\text { "request/token using/new token" }\end{array}$ \\
\hline
\end{tabular}

Fig. 1. Data Structures

\section{DESCRIPTION OF FAULT-TOLERANT AIGORITHM}

In this section, we describe the exact algorithm performed by an arbitrary node $i$. The exact algorithm is shown in Fig. 1 .

\subsection{Requesting Critical Section}

To request the critical section, a process increments its component in $v$ vector and sends the request with its $v$ vector to all the processes including itself. After then, it sets timeout and flag to "request". If it does not receive the token and "token_using" message within a timeout, it suspects the token loss as denoted by line 1-5 in algorithm of Fig. 2.

\subsection{Receiving a request of Critical Section}

On receiving a request, a process updates its $v$ vector with the maximum. After then, it appends the request to the myreqlist. If it has the token and does not use the critical section, then it checks for eligible requests and sends the token to one of the eligible processes. But if it is in the critical section, it sends "token_using" message to the requesting process as denoted by line 6-11

\subsection{Receiving a token}

On receiving the token, a process sends back an acknowledgment, which is efficient in case of the process failure. It receives the reqdone vector with the token. Processes keep reqdone updated through token passing. It sets inCS and havetoken to true. Then, it calls checkdone()procedure to delete the fulfilled request from the myreqlist. After then, it sends 'token_using" message to all the processes in myreqlist as denoted by line 12-17 in algorithm of Fig. 2.

\section{4. deleting the fulfilled requests from myreqlist}

To delete the fulfilled requests from the myreqlist, a process uses the reqdone vector. A request $w$ is considered fulfilled if $w . v$ is at most reqdone as denoted by line 18-21 in algorithm

\section{5 releasing critical section}

To release the critical section, a process sets in $C S$ to false. Then, it calls checkreq() procedure in order to check for eligible requests in the myreqlist to send the token as denoted by line 22-24 in algorithm of Fig. 2.

\subsection{Checking Eligible Requests in myreqlist}

To send the token, a process checks for eligible requests in the list (myreqlist). A request $w$ is eligible if $w \cdot v$ is at most reqdone, that is, there is no request that happened before $w$ and has not been fulfilled yet. Then, it deletes the eligible process from the myreqlest and the increments reqdone vector of the eligible process. It sends the token to one of the eligible processes and sets havetoken to false. If there is no eligible process to send the token, the process holds the token denoted by line $27-37$

\subsection{Receiving message that token has been occupied}

On receiving a "token_using" message, a process keeps flag to "request"and sets timeout. If it does not receive the token and "token_using" message within a timeout, it suspects the token loss. In our algorithm, if a process uses token, it sends a "token_using" message to the requesting process when it receives a request or the token. In addition, the token holding process periodically sends the "token_using" message to the processes in the myreqlist. As denoted by line 38-40 in algorithm of Fig 2 . 


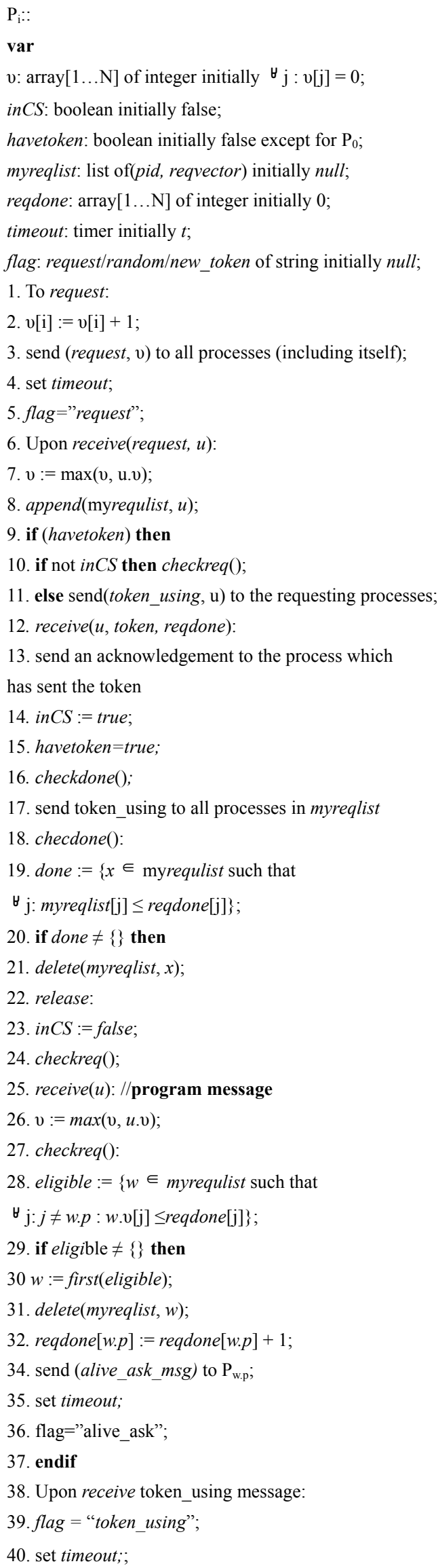

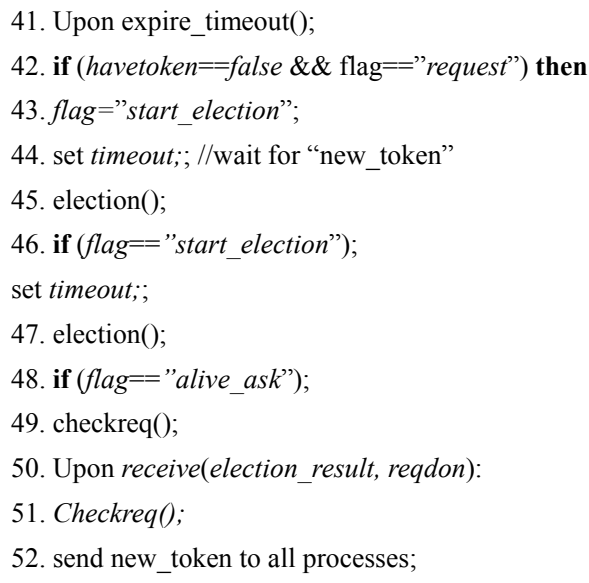

Fig 2. Proposed Algorithm

\subsection{Actions to be made upon timeout}

When timeout period has been elapsed, actions are made according to the value of flag. After sending a request (flag="request"), if Pi does not receive the token within a timeout period and "token_using" message periodically, it suspects the failure of the process with token. Then it starts election process.

After finishing the election process, Pi informs the elected process that it is the token generator. Then, it generates a new token and sends it to the next eligible process and "new_token"message to all processes to inform that a new token has been generated.

After setting flag to "start_election", if Pi does not receive "new_token" message within a timeout period, it suspects the failure again (during election, after election or after generating token) and restarts the election process Those procedures are denoted by line 41-49 in algorithm of Fig. 2 .

\subsection{Sending newly generated token}

If $\mathrm{Pi}$ is informed that it is the token generator, then it generates a new token and checks for the eligible process to send the token. In addition, it sends "new_token" message to all processes to inform that a new token has been generated as denoted by line 50-52 in algorithm of Fig 2 .

\section{PROOF OF CORRECTNESS}

In this section we prove that the algorithm ensures these three properties of mutual exclusion.

Theorem 1: At any instant, only one process has permission to use the critical section (safety property).

Proof: Because there is only one token in the system, no two processes hold the token simultaneously. Thus, the only one process holding the token can executes the critical section.

Theorem 2: Every requesting process eventually executes the critical section (liveness property).

Proof: A token request message of a process reaches other processes in finite time since communication links are reliable and thus message loss does not occur. Any request will be placed in the token reqlist in finite time since one of the 
processes will eventually have the token and every process maintains the records of all requests. Since there can be at most $\mathrm{N}-1$ requests made before the request of a process, the requesting process will eventually have the opportunity to execute the critical section after all the requests of other processes made before its request have granted.

Theorem 3: Different requests must be granted in the order they are made (fairness property).

Proof: Let $R_{i}$ be the request from a process $P_{i}$ and $R_{j}$ be the request from a process $P_{j}$. When Ri is made before $R_{j}$, we will prove that for these two requests, $P_{j}$ enters $C S$ after $P_{i}$ finishes CS.

In the algorithm (Fig. 1.), to send the token, a token holding process checks for the next eligible request. A request $w$ is eligible if there is no request that happened before $w$ and has not been fulfilled yet. If every component except $P_{j}$ 's in $v$ vector of $\mathrm{R}_{\mathrm{j}}$ is at most reqdone, $\mathrm{R}_{\mathrm{j}}$ is the next eligible request. If $R_{i}$ is not fulfilled yet, $P_{i}$ 's component in $v$ vector of $R_{j}$ is not at most reqdone.

Therefore, since $R_{j}$ can not be the next eligible request unless $R_{i}$ is not fulfilled, $P_{j}$ can not enter CS before $P_{i}$. Only $P_{j}$ can enter CS after $\mathrm{P}_{\mathrm{i}}$ finishes CS.

\section{SIMULATION AND PERFORMANCE ANALYSYS}

We, here, analyse the performance of proposed algorithm and simulate it in order to confirm if the algorithm works well on the real world. For performance analysis, we use simulating model as used in [7]. We use a distributed system with 10-50 processes(nodes). Each process makes request at random time and to make our simulation rather simple, we assume that there are no simultaneous requests from processes in the system at a time. Request arrival pattern at a process(node) is Poisson distribution. We assume that three different types of timeout such as "request/token_using/new_token" has the same value and we set it as $30 \mathrm{~ms}$. We also assume arbitrary process failures. For performance analysis, we consider the parameter LTS(latency to stable status) which means the latency from time that a process detect failed process to the time that system reach to the stable status. As showed in Table 1 and Fig.2, the result of the simulation shows us that the system does not be stopped by any kind of failures during execution. According to the type of timeout, latency time to the stable status varies and there is no formal pattern showed.

\section{CONCLUSIONS}

Table 1. Simulation Results

\begin{tabular}{|c|c|c|}
\hline $\begin{array}{c}\text { No. of } \\
\text { processes }\end{array}$ & $\begin{array}{c}\text { Type of timeout } \\
(30 \mathrm{~ms})\end{array}$ & $\begin{array}{c}\text { Latency to } \\
\text { stable status(sec) }\end{array}$ \\
\hline \multirow{4}{*}{10} & request & 0.91 \\
\cline { 2 - 3 } & token_using & 0.84 \\
\cline { 2 - 3 } & new_token & 1.27 \\
\hline \multirow{4}{*}{20} & request & 1.24 \\
\cline { 2 - 3 } & token_using & 1.19 \\
\cline { 2 - 3 } & new_token & 1.38 \\
\hline \multirow{4}{*}{30} & request & 1.85 \\
\cline { 2 - 3 } & token_using & 1.94 \\
\cline { 2 - 3 } & new_token & 2.38 \\
\hline \multirow{4}{*}{40} & request & 2.18 \\
\cline { 2 - 3 } & token_using & 2.03 \\
\cline { 2 - 3 } & new_token & 2.35 \\
\hline \multirow{3}{*}{50} & request & 3.49 \\
\cline { 2 - 3 } & token_using & 3.52 \\
\cline { 2 - 3 } & new_token & 3.94 \\
\hline
\end{tabular}

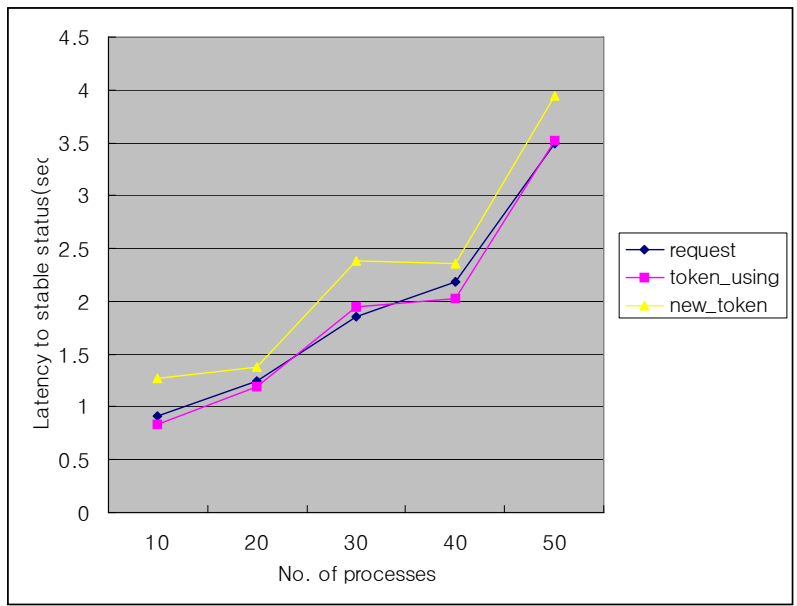

Fig. 2. Simulation results

In this paper, we proposed a new fault-tolerant distributed mutual exclusion algorithm in distributed system which assumes a fault-free system. To detect process failures, we used acknowledgements and timeout based re-transmission of messages which are commonly used techniques for fault detection in a distributed system.

As proved in section 6, proposed algorithm works properly against both node and network failures satisfying three properties of mutual exclusion. We can assume by

intuition that proper timeout value in our algorithm will vary according to the system environments, that is, the number of processes, and reliability of nodes and network etc. Future works include

simulation and performance analysis which will be expected to give us reference to deciding what are the proper values of timeout in corresponding system. 


\section{REFERENCES}

[1] Vijay K. Garg, Elements of Distributed Computing, A John Wiley \& Sons Inc., Publication, New York, 2002

[2] D. Agrawal and A. El Abbadi, An efficient solution to the distributed mutual exclusion problem, in Proc. 8th ACM Symposium on Principles of Distributed Computing, pp. 193-200, 1989

[3] G.Ricart and A.K.Agrawala, An optimal algorithm for Mutual Exclusion in Computer Networks, Commu nications of the ACM, 1981

[4] M. Singhal, A dynamic information structure for mutual exclusion algorithm for distributed systems, IEEE Transactions on Parallel Distributed Systems, 3(1):121125, 1992

[5] M. Maekawa, A Square Root N Algorithm for Mutual Exclusion in Decentralized Systems, ACM Transactions on Computer Systems(TOCS), 324):145-159, May 1985

[6] I. Suzuki and T. Kasami,A distributed mutual exclusion algorithm, ACM Transactions on Computer Systems (TOCS), 3(4):344-349, 1985

[7] M. Singhal, A Heuristically Aided Algorithm for Mutual Exclusion in Distributed Systems, IEEE Transactions on Computers, vol. 38, no. 5, pp. 651-662, May 1989

[8] Quazi Ehsanul Kabir Mamun and Hidenori Nakazato, A New Token Based Protocol for Group Mutual Exclusion in Distributed Systems, IEEE Proceedings of the fifth International Symposium on Parallel and Distributed Computing, 2006

[9] K. Raymond, A tree-based algorithm for distributed mutual exclusion, ACM Transactions on Computer Systems (TOCS), 7(1):61-77, 1989

[10] J. Sopena, L Arantes, M Bertier and P Sens. A FaultTolerant Token-Based Mutual Exclusion Algorithm Using a Dynamic Tree. In EuroPar 2005, Lisboa, Portugal, September 2005. LNCS, 2005.

[11] S. Mishra and P.K. Srimani Fault-tolerant mutual exclusion algorithms. Journal of Systems Software, 11(2):111-129, February 1990.

[12] M. Singhal, A heuristically-aided algorithm for mutual exclusion in distributed systems, IEEE Transactions on Computers, vol. 38, No.5, May 1989, pp. 651-662.

[13] Chang, M. Singhal, and M. Liu, A fault tolerant algorithm for distributed mutual exclusion, in Proc. of 9th IEEE Syrup. On Reliable Dist. Systems, 1990 pp. 146-154.

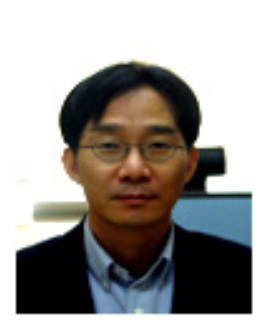

Yoon Kim [Regular member]

He received the B.S.,in mechanical engineering from Hanyang university, Korea in 1982 and received M.S. in computer science from Stevens Institute of Technology, USA and received $\mathrm{PhD}$. in computer science from Chungbuk National University. Currently he is a professor of Korea National College of Welfare. 